\title{
Diabolo Optical Antenna for Enhancing and Confining Electric Field Resonance
}

\author{
Mohammed Nadhim Abbas \\ Electrical Engineering Department, \\ College of Engineering, University of Baghdad, \\ Baghdad, Iraq
}

\author{
Hadi K. Shamkhi \\ Electrical Engineering Department, \\ College of Engineering, \\ University of Baghdad, Baghdad, Iraq
}

\begin{abstract}
In this paper, the electric resonance characteristics of narrow waist diabolo shape gold metal bar nanoantenna have been investigated. These metal nanostructures induce surface plasmon polration (SPP) and localized surface plasmon resonance (LSPR) leading to enhance the absorption and the scattering cross sections characteristics of the antenna. Investigation is done using 3D model Finite Element Method (FEM) numerical simulations and dipole oscillator model. From simulation work, it is found that the relationship between the antenna length and the resonance wavelength is approximatly linear. Also, it is found that as the diabolo antenna waist get narrower, the power dissipation decreases and slightly the resonance wavelength shifted to the higher values. The investigation shows that the electric field amplified in the extremities of the diabolo antenna while the magnetic field $90^{\circ}$ phase shifted from the electric field and amplified in the waist (middle) of the antenna.
\end{abstract}

\section{General Terms}

Antenna, optical antenna, nanoparticles.

\section{Keywords}

Plasmonics, Surface plasmon polration, Localized surface plasmon resonance, Dipole oscillator model, Absorption cross section, Scattering cross section.

\section{INTRODUCTION}

Optical antenna is very promising solution to overcome the limitations of bandwidth and fundamental speeds in modern electronic devices [1]. Depending on their shapes metal nanoantenna able to support localized surface plasmon resonance (LSPR) and excite confined electromagnetic fields on surfaces and wedges [2]. Optical antenna amplifies the electric and or magnetic fields which are useful for many application areas. The typical examples include spectroscopy $[3,4]$, sensing $[5,6]$, nanolithography $[7,8]$, and photodetection $[9,10]$.

Optical antennas with various configurations have been investigated in the last few years. The nonradiating character of LSPR in silver nanowire gives raise to interested values of surface plasmon propagation length and nanowire extremities reflectivity of about $10 \mu \mathrm{m}$ and $25 \%$ respectively [11]. An array of nanowire gives the ability to wide shift in resonance wavelength, also more confined electromagnetic fields depending on their number and spacing between them [12]. Nanoapertures in ultra-thin silver film radiates at optical frequencies and behaves as an optical antenna array. Its radiating shows a strong directivity depending on two factors, the in-plane rotation of aperture array, and on the polarization and incidence angle of exciting light [13]. Bi-harmonic metallic grating can enable strong SPP excitation and forms a band gap for the propagation of the surface plasmon portion, also enables the control of the plasmonic band gap structure [14]. Electrically connected resonant optical antenna offers the realization of highly efficient nanoscale electro- plasmonic devices that rely on a combination of electric fields and local near-field intensity enhancement [15]. In spectroscopies, coupled gold nanorods structures could be critical in use. Its near fields in a gap can be much higher than the extremities of isolated structures [16]. In nanorods structure, rod length, interred gap, and rod radius are each important for responding to the excitation incident light. Optical dimer antennas also have been investigated. It is near-field and far-field properties are determined by geometry design at the gap and the global shape of the antenna metal bar respectively. A hybrid dimer antenna, which is mixed between bowtie and rod metal bar, gives 30\% more benefit to near and far fields [17].

The electromagnetic response of the Diabolo optical antenna has been investigated using (FEM) numerical simulations, which consists of two gold metal triangles connected physically at the tips. The simulation gives more reliable solutions than other similar ones, because it is done with the 3D model. Plane wave propagated in the $z$-direction is used to excite the model at normal incidence. Bowtie antenna amplified electric field in the gap while diabolo antenna amplified magnetic field in the middle. The triangle shape lead to differentiating in electron motions along the antenna. At extremities where electrons motion approaches zero, the results show strong electric field enhancement, while in the middle electrons motion becomes extremely fast and present amplified magnetic field. Investigations show the distribution of total absorbed power on the diabolo geometry, which gives sense to how the geometry modifications will affect the antenna electromagnetic response.

\section{DIABOLO OPTICAL ANTENNA}

\subsection{Study model}

Study model consists a three dimensional gold metal bar diabolo shape optical antenna on a sapphire dielectric substrate (see figure 1). The antenna length is $1 \mu \mathrm{m}$ in the $x$ direction. The width at the two compatible ends is $300 \mathrm{~nm}$ in the $y$ direction. The waist of the antenna is $30 \mathrm{~nm}$ and the height is $60 \mathrm{~nm}$ in the $z$ direction. Using FEM numerical algorithm, the resonance properties of diabolo optical antenna are investigated. The simulation region cross section is 

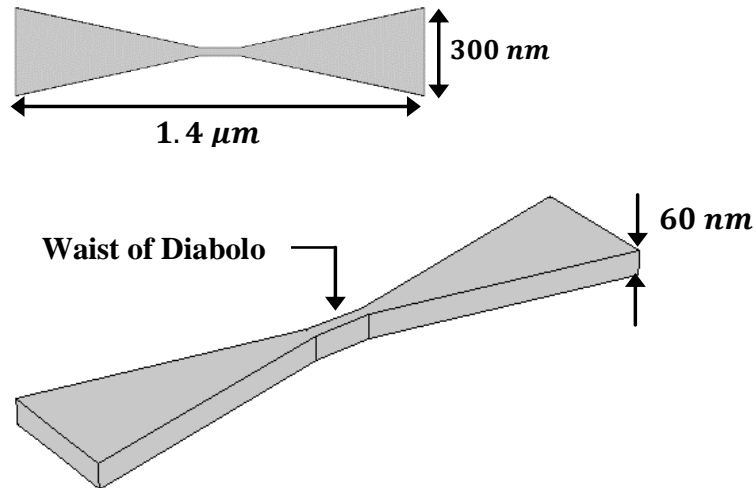

Fig 1: 3D diabolo narrow waist gold bar optical antenna with it is top view.

$3 \times 3 \mu m^{2}$ in the $x y$ direction and the height is $4 \mu \mathrm{m}$ in the $z$ direction. The air is considered to occupy the $z>0$ region and the sapphire occupies the $z<0$ region. The optical constants of gold and sapphire are implemented in the model according to Palik's handbook [18]. Plane TE-polarized electromagnetic wave is incident on a gold diabolo on a dielectric substrate at normal incidence. With the diabolo antenna placed on a substrate, the analytical expression for the background field becomes more complicated. It needs to be corrected the superposition of the incident and reflected wave in the free space domain, and a transmitted wave in the substrate. A simple and general way to avoid deriving and entering the analytical background field is to use a full field solution of the problem without the antenna. To achieve this full field solution, the simulation is set up with two Port conditions. One defines the incident plane wave and allows for specular reflection. The other absorbs the transmitted plane wave. Perfectly matched layers (PML) with one to fourth thickness of the incident light wavelength surrounding the study volume in every direction. PML reflect no waves and hence ensures convergence solution.

\subsection{Dipole resonance model}

It can be understood the electromagnetic wave behavior of the antenna by using the dipole oscillator model. The balance equation consists of exciting power, internal damping force and, attractive force between electron and atomic nucleus [1923]. In this model, the averaged power dissipated $P$ the optical antenna is related to the complex conjugate

force applied to the electron $F^{*}(w)$ as

$$
\begin{aligned}
& F(w)=-q L \frac{d^{2} x(w, t)}{d t^{2}} \\
& P(w)=F^{*}(w)\left[j w x_{0}(w)\right]
\end{aligned}
$$

Where $q$ is the electron charge, $L$ is the self-induced inductance force and $x(w, t)$ is the electron motion. The current density $\left(A / \mathrm{m}^{2}\right)$ is the first degree derivative of electron motion $(d x(w, t) / d t)$.

The absorption cross section $C_{a b s}$ and the scattering cross section $C_{s c a}$ can be written as

$$
\begin{aligned}
& C_{a b s}=P_{a b s} / I_{0} \\
& C_{s c a}=P_{s c a} / I_{0}
\end{aligned}
$$

Where $P_{a b s}$ is the volume integration of the power loss density in the diabolo antenna and, $P_{s c a}$ is the surface integration of the pointing vector time the normal vector pointing outward from the nanodot.

\section{RESULTS AND DISCUSSIONS}

The electric field norm $(V / m)$ and magnetic field norm $(\mathrm{A} / \mathrm{m})$ are calculated along the interface of diabolo gold metal bar antenna with the sapphire substrate in the $x$ direction (see Figures 2.a, b and 3.a, b). It can be seen that the electric field norm maximum value at the extremities and decreased exponentially to the middle of the diabolo antenna. The enhancement of electric field in the edges is more than 6 times from that in the waist of the antenna. By means of Faraday's law of induction $(\nabla \times E=-\partial B / \partial t)$, the magnetic field norm induced and $90^{*}$ phase shifted from the electric field, where $E$ is electric field and $B$ is magnetic flux density. The enhancement of the magnetic field in the waist is more than 15 times that in the edges of the antenna.

The power dissipation along the interface between the Diabolo metal antenna and the dielectric substrate in the $x$ direction is also calculated (see figures $2, \mathrm{~d}$ and $3, \mathrm{~d}$ ). It can be seen that the curve of power dissipation represent the derivation of current density curve (see figures 2 , c and $3, \mathrm{c}$ ) multiplied by other factors as equation 2 explained. Total power dissipation density is the sum of electromagnetic power loss, resistive loss, surface loss and, magnetic loss. The focusing of power loss in the waist of antenna gives the geometry of the waist crucial rule in the antenna wave absorption property.

The electron motion is slow or zero in the extremities of optical antenna lead to amplification of the charge density, and increasing in the electric field according to Gauss's law.
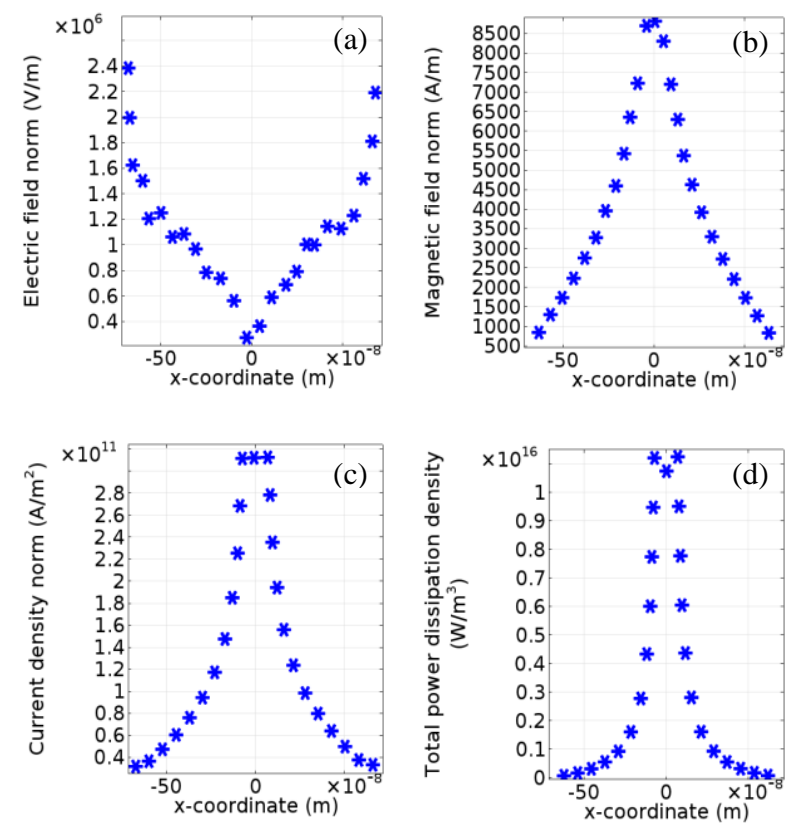

Fig 2: (a) Electric field norm, (b) Magnetic field norm, (c) Current density and, (d) Total power dissipation density versus $x$-coordinate along with the interface between diabolo metal antenna and dielectric substrate. 
(a)

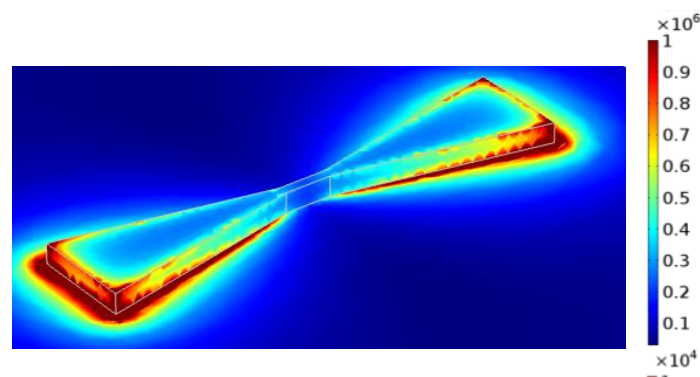

(b)

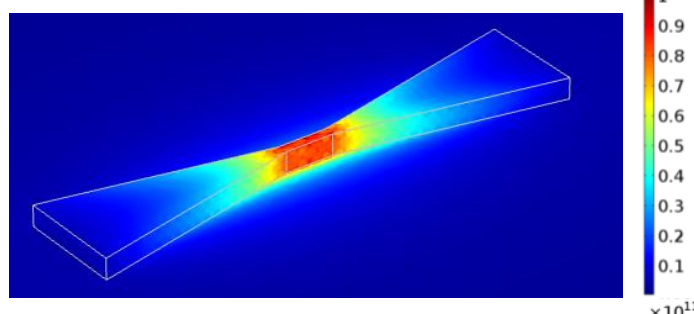

(c)

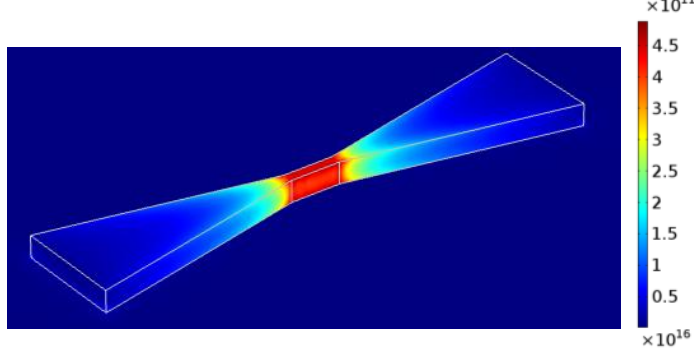

(d)

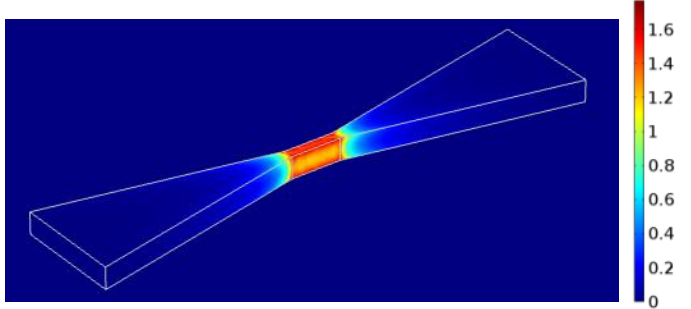

Fig 3: 3D image view with color legend for (a) Electric field norm, (b) Magnetic field norm, (c) Current density and, $d$. Total power dissipation density.

Gradually electron motion gets faster as the metal bar become narrower. At the waist of optical antenna, speed of electrons and current density maximized leads to amplification in the magnetic field according to Maxwell-Faraday equation $\nabla \times$ $B=\mu_{0}\left(J+\varepsilon_{0} \frac{\partial E}{\partial t}\right)$. Where $J$ is the current density, $\mu_{0}, \varepsilon_{0}$ is the permeability and the permittivity of free space respectively.

Absorption cross section and scattering cross section are calculated versus free space wavelength (see figure 4.a, b). The calculations is done for different antenna lengths starting with $400 \mathrm{~nm}$ and endend in $1400 \mathrm{~nm}$ with step size $200 \mathrm{~nm}$. There are two resonance modes can be observed, the fundamental resonance mode locate at longer wavelength while the high order resonance mode occur at shorter wavelength. In this paper, only the fundamental mode is discussed.

It can be seen that, as the metal bar length increased, there are monotonic redshift in the resonance wavelength in both the absorption cross section and the scattering cross section. The shifting is approximately linear with the antenna length and can be represented by $\lambda_{\text {Resonance }} \cong \frac{1}{8.75}$ L to $\frac{1}{4.6} L$. There are $4 \mu \mathrm{m}$ wavelength shift $(7.385 \mathrm{E} 13 \mathrm{~Hz})$ between the 0.4 - and
1.4- $\mu \mathrm{m}$-antenna lengths. This redshift is accounted for by the fact that it takes a longer time for the electrons to traverse a long metal bar antenna, yielding a lower resonance frequency, and therefore, a longer wavelength.

The area of incident radiation that interacts with the antenna or the antenna aperture is rapidly doubled with the increasing in the antenna length. As a result, the absorption cross section and the scattering cross section maximum values raised by 8.5 and 6 times respectively (see figure $4 . a, b$ ).

The influences of changing the antenna waist width on the absorption and scattering cross sections are investigated (see figure 5.a, b). The absorption cross section versus the wavelength for diabolo metal bar antenna with different waist widths $w=30 \mathrm{~nm}, 60 \mathrm{~nm}$ and $110 \mathrm{~nm}$ (see figure 5.a, b). The peak absorption wavelengths are $5 \mu \mathrm{m}, 4.76 \mu \mathrm{m}$ and, , $4.59 \mu \mathrm{m}$ respectively. It can be seen that, the absorption cross section peak decreased from $0.73 \mu \mathrm{m}^{2}$ at $w=30 \mathrm{~nm}$ to $0.425 \mu \mathrm{m}^{2}$ at $w=120 \mathrm{~nm}$. The waist region absorbs most of the power that dissipated in the diabolo antenna. As the antenna waist become wider, the speed of the electrons motion become slower, reducing current density and, decreasing in absorption power density, which is cleared from dipole oscillator mode (see equation 2).
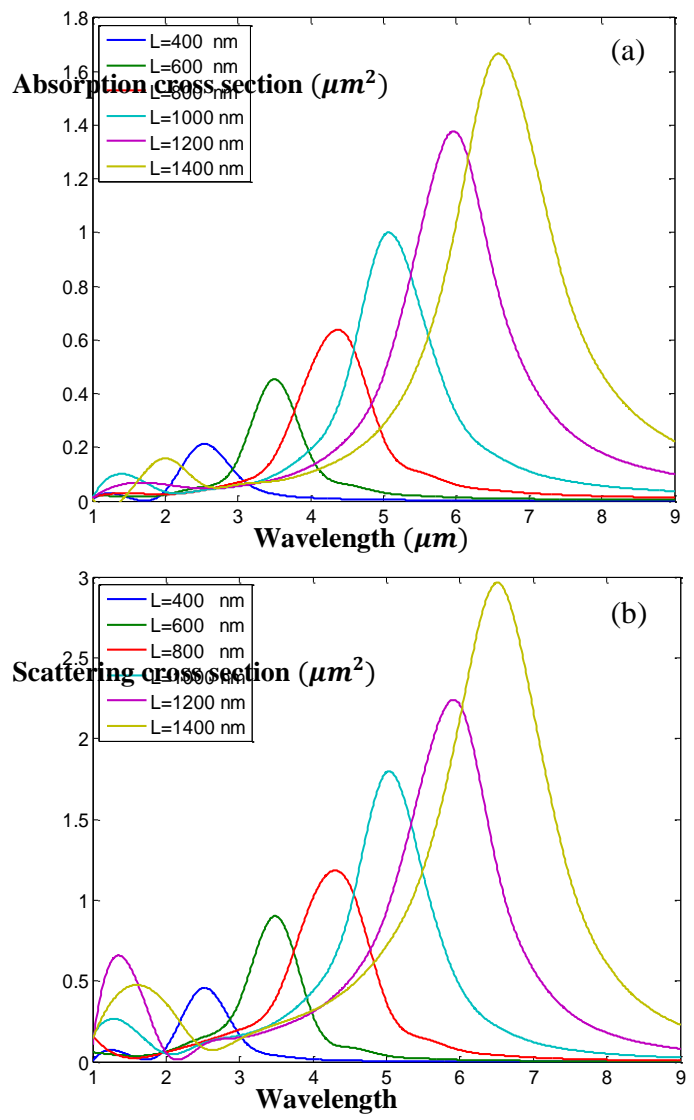

Fig 4: a, b Absorption cross section and scattering cross section versus wavelength for different antenna waist widths respectively.

Fig 5: a, b Absorption cross section and scattering cross section versus wavelength for different antenna waist widths respectively. 

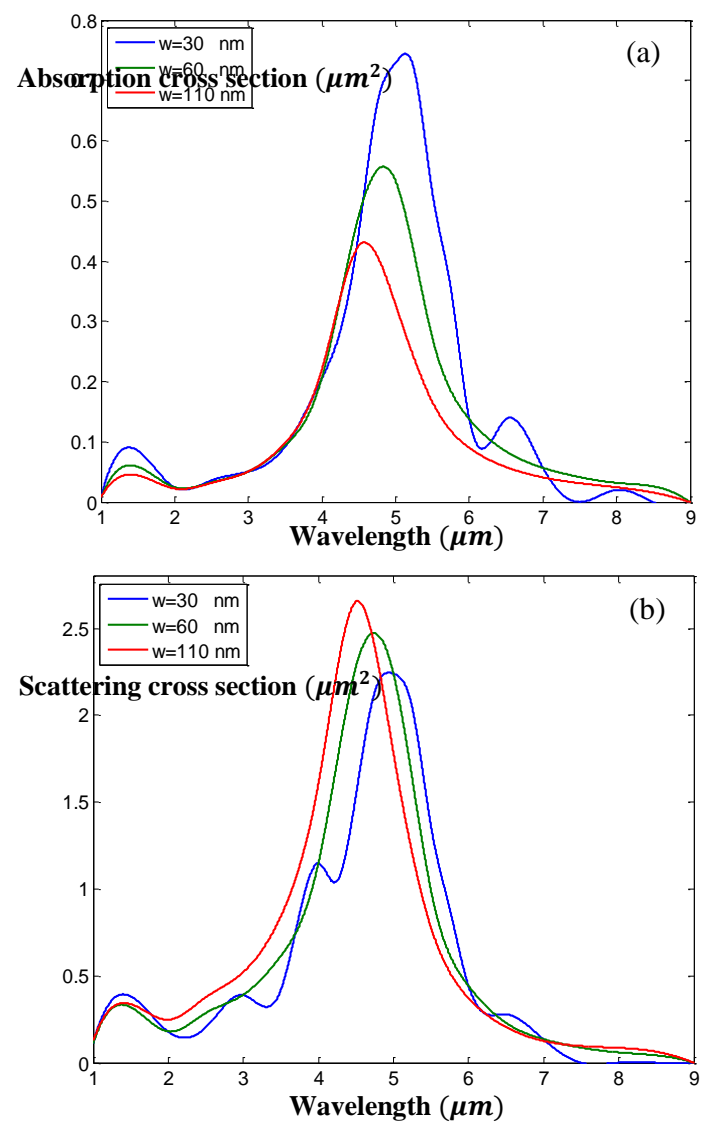

The scattering cross section versus the wavelength for metal bar waist widths of $w=30 \mathrm{~nm}, 60 \mathrm{~nm}$ and, $110 \mathrm{~nm}$. The peak scattering wavelength are $5.1 \mu m, 4.78 \mathrm{~nm}$ and $4.52 \mathrm{~nm}$ respectively. The scattering cross section peak decreased from $2.65 \mu m^{2}$ at $w=60 \mu m$ to $2.24 \mu m^{2}$ at $w=30 \mathrm{~nm}$.

\section{CONCLUSION}

In this paper, the electric field resonance of the diabolo optical antenna is investigated, considering impact of changing the antenna length and the antenna waist width. From simulation results, it is found that the charge density is maximum at the extremities cause the electric field to be enhanced and decayed exponentially to the middle of the antenna. Oppositely, the current density maximized in the waist leading to enhance the magnetic field which is decayed relatively slow toward the extremities of the antenna. It is also noticed that the power dissipation happened mostly in the waist which is equal to the current density derivation multiplied by frequency, inductance and other factors. Simulations shows, as the antenna length increased, the absorption cross section and the scattering cross sections peaks increased exponentially and the resonance wavelength shifts to higher values. Finally, the narrowness of the antenna waist increased the peaks of absorption cross section and decreased that of the scattering cross section, while both cross sections peaks shifted to lower wavelength values.

\section{REFFRENCES}

[1] D.K. Gramotnev, S.I. Bozhevolnyi, "Plasmonics beyond the diffraction limit", Macmillan Publishers Limited, 2010, Nature Photonics.
[2] T. Grosjean, M. Mivelle, F. I. Baida, G. W. Burr, and U. C. Fischer, " Diabolo Nanoantenna for Enhancing and Confining the Magnetic Optical Field", ACS Publications, 2011, nano letters.

[3] A. Pucci, F. Neubrech, D. Weber, S. Hong, T. Toury, and M. L. de la Chapelle, "Surface enhanced infrared spectroscopy using gold nanoantennas" , 2010, Phys. Stat. Solidi.

[4] L. Billot, M. Lamy de la Chapelle, A. S. Grimault, A. Vial, D. Barchiesi, J. L. Bijeon, P. M. Adam, and P. Royer, "Surface enhanced Raman scattering on gold nanowire arrays: evidence of strong multipolar surface plasmon resonance enhancement" , 2006, Chem. Phys. Lett.

[5] J. N. Anker, W. P. Hall, O. Lyandres, N. C. Shah, J. Zhao, and R. P. Van Duyne, "Biosensing with plasmonic nanosensors" , 2008, Nat. Mater.

[6] P. Bharadwaj, P. Anger, and L. Novotny, "Nanoplasmonic enhancement of single-molecule fluorescence", 2007, Nnotechnology.

[7] L. Wang, S. M. Uppuluri, E. X. Jin, and X. Xu, "Nanolithography using high transmission nanoscale bowtie apertures", 2006, Nano Lett.

[8] A. Sundaramurthy, P. J. Schuck, N. R. Conley, D. P. Fromm, G. S. Kino, and W. E. Moerner, "Toward nanometer-scale optical photolithography: Utilizing the near-field of bowtie optical nanoantennas" , 2006, Nano Lett.

[9] J. A. Schuller, E. S. Barnard, W. Cai, Y. C. Jun, J. S. White, and M. L. Brongersma, "Plasmonics for extreme light concentration and manipulation," 2010, Nat. Mater.

[10] J. Aizpurua, G.W. Bryant, L.J. Richter, and F. J. García de Abajo, "Optical properties of coupled metallic nanorods for field-enhanced spectroscopy", 2005, Phys. Rev

[11] H. Ditlbacher, A. Hohenau, D.Wagner, U. Kreibig, M.Rogers, F.Hofer, F.R. Aussenegg, and J.R. Krenn, "Silver Nanowires as Surface Plasmon Resonators", 2005, Phys. Lett.

[12] E. Cubukcu, N.Yu, E.J. Smythe, L.Diehl, K.B. Crozier, and F.Capasso, "Plasmonic Laser Antennas and Related Devices", 2008, IEEE J. Sel. Topics Quantum Electron.

[13] Z. J. Zhang, R. W. Peng, Z. Wang, F. Gao, X. R. Huang, W. H. Sun, Q. J. Wang, and Mu Wang, " Plasmonic antenna array at optical frequency made by nanoapertures", 2008, Appl. Phys. Lett.

[14] A.Kocabas, S.S. Senlik, and A.Aydinli, "Plasmonic band gap cavities on biharmonic gratings", 2008, Phys. Rev. Lett.

[15] J.C. Prangsma, J.Kern, A.G. Knapp, S.Grossmann, M.Emmerling, M.Kamp, and B.Hecht, "Electrically Connected Resonant Optical Antennas", 2012, Nano Lett

[16] J. Aizpurua, G.W. Bryant, L.J. Richter, and F. J. García de Abajo, " Optical properties of coupled metallic nanorods for field-enhanced spectroscopy", 2005, Phys. Rev.

[17] W. Ding, R. Bachelot, R. Espiau de Lamaestre, D. Macias, A.L. Baudrion, and P. Royer, "Understanding 
near/far-field engineering of optical dimer antennas through geometry modification", 2009, Optics Express

[18] E.D. Palik, Handbook of Optical constants of solids (Academic press, 1998).

[19] Z.Pan, J.Guo, "Enhanced optical absorption and electric field resonance in diabolo metal bar optical antennas", 2013, Opt. Express.

[20] M. A. Kats, N. Yu, P. Genevet, Z. Gaburro, and F. Capasso, "Effect of radiation damping on the spectral response of plasmonic components", 2011, Opt. Express.
[21] B. M. Ross and L. P. Lee, "Comparison of near- and farfield measures for plasmon resonance of metallic nanoparticles", 2009, Opt. Lett.

[22] J. D. Jackson, Classical Electrodynamics 3th ed. (Wiley, 1998).

[23] H.Raether, Surface Plasmons on Smooth and Rough Surfaces and on Gratings, (Springer, 1986). 